\title{
ARTIGOS
}

\section{AVALIAÇÃO DO DESEMPENHO ESTRUTURAL DE PAREDES DE MADEIRA LAMINADA COLADA}

\section{STRUCTURAL PERFORMANCE EVALUATION OF GLUED LAMINATED TIMBER WALLS}

\author{
EDGAR VLADIMIRO MANTILLA CARRASCO, Dr.| UFMG \\ CYNARA FIEDLER BREMER, Dra. | UFMG \\ JUDY NORKA RODO DE MANTILLA, Dra. | UFMG
}

\begin{abstract}
RESUMO
O objetivo deste trabalho é determinar o desempenho estrutural de paredes de madeira laminada colada utilizando a norma brasileira NBR 15575 (2013). Foram confeccionadas 5 paredes com seção transversal de $9 \mathrm{~cm} \times 140$ $\mathrm{cm}$ e altura de $260 \mathrm{~cm}$, sendo 3 de Eucalyptus cloeziana e 2 de Eucalyptus grandis. O adesivo utilizado foi a base de resina resorcinol formaldeído. Antes da realização dos ensaios foi feita uma caracterização física e mecânica da madeira. As paredes foram montadas a partir da união de 5 vigas de madeira laminada colada de $52 \mathrm{~cm}$ de comprimento. A união foi feita por meio do sistema macho-fêmea utilizando o adesivo. Foram realizados os ensaios de compressão simples, corpo mole e corpo duro, conforme especificações da NBR 15575 (2013). As condições de contorno adotadas foram: apoios fixos nas duas extremidades. A instrumentação foi constituída de uma célula de carga e três transdutores de deslocamento. A aplicação da carga foi monotônica até atingir carga máxima. Os resultados dos ensaios de compressão simples e dos ensaios de impacto de corpo mole e de corpo duro realizados nas paredes de madeira laminada colada indicam um elevado desempenho estrutural e, portanto, atendem os critérios de desempenho especificados pela NBR 15575 (2013).
\end{abstract}

PALAVRAS CHAVE: Madeira Laminada Colada; Paredes; Avaliação da conformidade

\begin{abstract}
The objective of this work is to determine the structural performance of glued laminated timber walls using the Brazilian standard NBR 15575 (2013). Five walls with cross-section of $9 \mathrm{~cm} \times 140 \mathrm{~cm}$ and height of $260 \mathrm{~cm}$ were made, 3 of these walls of Eucalyptus cloeziana and 2 of Eucalyptus grandis. The adhesive used was resorcinol formaldehyde resin base. A physical and mechanical characterization of the wood was performed before the tests. The walls were assembled from the union of 5 glued laminated timber beams of $52 \mathrm{~cm}$ in length. The union was made through the male-female system, using adhesive. Simple compression, soft body and hard body impact tests were performed, according to the specifications of NBR 15575 (2013). The boundary conditions were fixed at both ends. The instrumentation consisted of a load cell and 3 displacement transducers. The load application was monotonic until reaching maximum load. The results of the simple compression tests and the soft body and hard body impact tests indicate high structural performance of the glued laminated timber walls, and, therefore, meeting the specifications of NBR 15575 (2013).
\end{abstract}

KEY WORDS: Glued Laminated Timber;Walls;Performance evaluation 


\section{INTRODUÇÃO}

O surgimento dos painéis de madeira ocorreu com o isolamento da Alemanha durante a Segunda Guerra Mundial, quando o país se viu diante de dificuldades que fomentaram o aparecimento de novas tecnologias. Uma delas foi o desenvolvimento de painéis de madeira aglomerada, na década de 1940, como forma de empregar os resíduos madeireiros disponíveis, em um cenário de escassez de madeira com características compatíveis com a produção dos compensados (Iwakiri, et al, 2005,Macedo, et al, 2015 e Lima, et al, 2020). A partir da década de 1980, as indústrias de base florestal no Brasil passaram por uma grande transformação em termos de matéria-prima, produtos e processos produtivos. A base da matéria-prima madeira, que era quase que totalmente oriunda de florestas nativas, passou a ser substituída por florestas plantadas de rápido crescimento, especialmente a de pinus e eucalipto. (ABIMCI, 2004 e lwakiri, et al, 2007).

O compensado estrutural é classificado como um painel multilaminado, colado com resinas à prova d'água, para uso em condições cíclicas de alta e baixa umidade relativa, eventualmente em ação direta com a água, de uso exterior. A sua aplicação se destina principalmente ao setor de construção civil e para embalagens (Baldwin, 1995 e Iwakiri, et al, 2007).

O painel de Oriented Strand Board (OSB) é reconstituído de flocos de madeira, parcialmente orientados, com a incorporação de adesivo à prova d'água e consolidados por meio de prensagem a quente. As chapas de OSB são produtos utilizados para aplicações estruturais, como suportes de piso e forro, componentes de vigas, estrutura de móveis e embalagens, competindo com o compensado estrutural. Por ser menos exigente do que o compensado na qualidade da matéria prima, o OSB está substituindo o mesmo em muitas aplicações (Cabral, et al, 2006).

Os painéis de madeira laminada colada cruzada (MLCC) denominados também de painéis CLT (Cross Laminated Timber), são formados por lâminas de madeira com grandes dimensões arranjadas ortogonalmente e unidas com adesivo estrutural sob alta pressão. $O$ sistema de laminação cruzada possibilita ao CLT trabalhar como um elemento estrutural rígido e autoportante (Amorim, et al, 2017, Sikora, et al, 2016 e Kolton, et al, 2020).

Caso as lâminas sejam dispostas paralelamente umas às outras tem-se a madeira laminada colada (MLC), fabricada a partir de pequenas placas de madeira planas ou lamelas coladas com adesivos estruturais. É capaz de formar peças maiores com propriedades mecânicas que são mais resistentes que as tábuas de madeira originais.
O material pode ter uma disposição homogênea, onde todas as laminações são da mesma classe de resistência, ou combinadas, onde as laminações externas são de uma classe de maior resistência. A MLC pode compor vigas estruturais, colunas, elementos de treliça ou paredes estruturais (Building and Construction Authority, 2018).

A norma brasileira de Desempenho, NBR15575 (2013), nas suas partes 2 e 4 , traz os requisitos necessários para os sistemas de vedações internas e externas das edificações habitacionais. Nelas são descritos os níveis de aceitação, conforme os resultados dos ensaios realizados (deslocamentos, fissuras e falhas). São previstos ensaios de compressão, de impacto de corpo mole e também de corpo duro. A modelagem matemática do comportamento conjunto para a resistência mínima de projeto prevê ensaios destrutivos, com pelo menos dez etapas de carga, com repetição para 3 modelos geométricos idênticos em escala real e com traçado de diagramas carga $\mathrm{x}$ deslocamento.

Neste trabalho foram analisadas paredes de MLC, formadas por vigas empilhadas, conectadas entre si por encaixes macho-fêmea, sob as premissas da norma de desempenho. Foram realizados os ensaios de compressão simples, de impacto de corpo mole e também de corpo duro.

\section{MATERIAIS E MÉTODOS}

A metodologia utilizada nos ensaios dos elementos estruturais foi orientada por normas brasileiras. Os ensaios dos protótipos foram realizados no LAEES - Laboratório de Análise Experimental de Estruturas. Os ensaios de caracterização mecânica da madeira foram realizados no Laboratório de Ensaios Mecânicos - LACAM. Os ensaios de caracterização física foram realizados no Laboratório de Caracterização Física - LACAF. Todos estes laboratórios são do Departamento de Engenharia de Estruturas DEES - da Universidade Federal de Minas Gerais - UFMG.

\subsection{Detalhes da fabricação das paredes de MLC}

A espessura das lâminas utilizadas na fabricação dos painéis estruturais de MLC variou em torno de $2,9 \mathrm{~cm}$. As emendas longitudinais utilizadas foram do tipo finger joint, permitindo aumentar os comprimentos das peças e um maior aproveitamento da madeira por meio da eliminação de defeitos. As dimensões destes entalhes variaram em função da espessura da lâmina adotada na fabricação das peças de MLC. Foi utilizado o adesivo estrutural resistente à umidade à base de resina resorcinol, Cascophen (fabricado pela Borden Química Ltda.), com tempo de pega de aproximadamente 1 hora. Cuidado especial foi dado à preparação da superfície das lâminas antes da 
aplicação do adesivo. Para se obter a máxima resistência da linha adesiva, as superfícies das lâminas foram preparadas de forma a se ajustarem com perfeição. Observou-se que madeiras de elevadas densidades requerem menos adesivo que as espécies de baixa densidade ou porosas. As lâminas receberam o adesivo em suas superfícies e depois foram levadas às prensas.

Cada parede de MLC foi constituída por 5 placas, com as dimensões e ligações do tipo macho-fêmea, apresentadas na Figura 1.
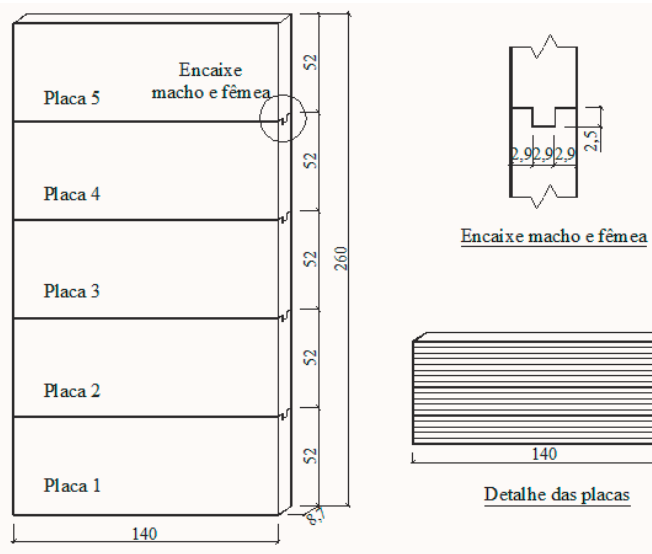

Vista da parede de MLC

Figura 1 - Parede de MLC

Fonte: 0 s autores

\subsection{Ensaios realizados}

\subsubsection{Caracterização física e mecânica da madeira}

Foi feita uma caracterização física e mecânica da madeira, segundo os preceitos da NBR7190 (1997) - Projeto de Estruturas de Madeira, em seu anexo $B$, nomeadamente: densidade aparente, resistência à compressão, modulo de elasticidade, resistência ao cisalhamento da madeira e do adesivo.

\subsubsection{Ensaios nas paredes de $M L C$}

A Norma ABNT 15575-4 (2013) traz em seu texto a recomendação de que painéis estruturais pré-fabricados devem ser ensaiados nas mesmas condições do emprego em obra, com altura prevista para o pé-direito e largura mínima de 1,20 m ou de 5 vezes a espessura, para paredes monolíticas. São previstos por esta norma ensaios de compressão simples, de impacto de corpo mole e de corpo duro. O comportamento conjunto para a resistência mínima de projeto prevê ensaios destrutivos, com pelo menos dez etapas de carga, com repetição para 3 modelos geométricos idênticos em escala real e com traçado de diagramas carga $x$ deslocamento.
O objetivo destes ensaios foi reproduzir paredes geralmente empregadas em habitações sociais. Desta forma, foram confeccionadas 5 paredes conforme dimensões apresentadas na Figura 1, sendo 3 paredes de Eucalyptus cloeziana e 2 de Eucalyptus grandis. Para o atendimento às condições de carregamento foram seguidas as prescrições da NBR7190 (1997). Nos ensaios de impacto de corpo mole, de corpo duro e de compressão simples, as condições de contorno adotadas foram baseadas nas condições verificadas na prática (rotuladas nas extremidades).

A carga na borda superior das paredes foi aplicada por um cilindro hidráulico e distribuída por meio de uma viga metálica. Nos ensaios de impacto de corpo mole e de corpo duro a carga distribuída (q) foi de aproximadamente 7000 N/m, com o intuito de se caracterizar o carregamento típico em uma parede. Os ensaios realizados nos protótipos das paredes estão apresentados na Tabela 1.

\begin{tabular}{|l|l|l|}
\hline $\begin{array}{l}\text { Espécie utilizada } \\
\text { na fabricação } \\
\text { dos elementos }\end{array}$ & $\begin{array}{l}\text { Número de } \\
\text { elementos } \\
\text { ensaiados }\end{array}$ & $\begin{array}{l}\text { Espécie utilizada na fa- } \\
\text { bricação dos elementos }\end{array}$ \\
\hline \multirow{2}{*}{$\begin{array}{l}\text { Eucalyptus } \\
\text { cloeziana }\end{array}$} & 3 & Compressão simples \\
\cline { 3 - 3 } & & $\begin{array}{l}\text { Resistência ao impacto } \\
\text { de corpo mole }\end{array}$ \\
\cline { 3 - 4 } & & $\begin{array}{l}\text { Resistência ao impacto } \\
\text { de corpo duro }\end{array}$ \\
\hline \multirow{3}{*}{ Eucalyptus grandis } & 2 & Compressão simples \\
\cline { 3 - 4 } & & $\begin{array}{l}\text { Resistência ao impacto } \\
\text { de corpo mole }\end{array}$ \\
\cline { 3 - 4 } & & $\begin{array}{l}\text { Resistência ao impacto } \\
\text { de corpo duro }\end{array}$ \\
\hline
\end{tabular}

Tabela 1 - Ensaios realizados nas paredes de MLC Fonte: 0 s autores

\subsubsection{Ensaio de compressão simples}

Depois de a parede montada e posicionada no pórtico, prosseguiu-se com a instrumentação. Os ensaios de compressão simples nas paredes foram realizados em um pórtico de reação com capacidade de 1000 kN, equipado com um cilindro hidráulico controlado por uma bomba manual para a aplicação do carregamento medido por uma célula de carga com capacidade de 500 kN. O encurtamento da parede foi monitorado, durante o ensaio, por dois transdutores de deslocamento DT (do termo em inglês Displacement Transducer) instalados nas laterais da parede, DT-1 e DT-2, e os deslocamentos horizontais (flechas) foram monitorados por um DT posicionado no meio do terço superior (DT-3), conforme prescrições da NBR15575 (2013), Figura 2. 


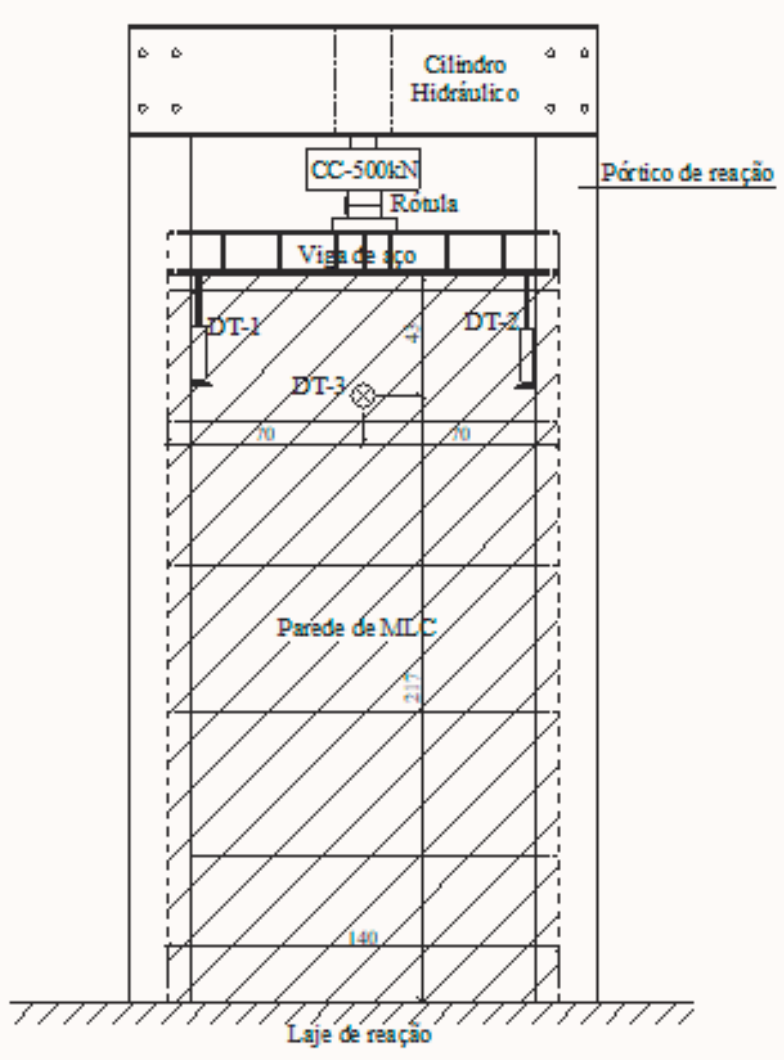

Figura 2 - Instrumentação do ensaio de compressão simples da parede (dimensões em cm) Fonte: 0 s autores

\subsubsection{Ensaios de impacto de corpo mole e de corpo duro}

Para a realização dos ensaios de impacto de corpo mole, depois de montado e posicionado o protótipo no pórtico de ensaio, conforme a Figura 3, um saco de areia com massa de $40 \mathrm{~kg}$ foi posicionado tendo o seu centro tangenciando o centro da parede. O ensaio consistiu em suspender o saco de areia, por diferentes alturas, e abandoná-lo em queda livre na direção à parede. Devido às características construtivas e às condições de vinculação das paredes, que são idênticas tanto para um carregamento aplicado da direita para a esquerda, quanto da esquerda para a direita, fez-se o ensaio em apenas uma direção. Já para a realização dos ensaios de impacto de corpo duro, depois de montado o corpo-de-prova, foram utilizadas duas esferas maciças de aço, uma com massa de $500 \mathrm{~g}$ e outra com massa de $1000 \mathrm{~g}$, para a imposição dos impactos na parede. Cada uma das esferas foi suspensa por um fio, tendo sua face tangenciando a parede quando em repouso. $O$ ensaio consistiu em levantar cada esfera por diferentes alturas, e abandoná-la em queda livre na direção da parede, Figura 4.

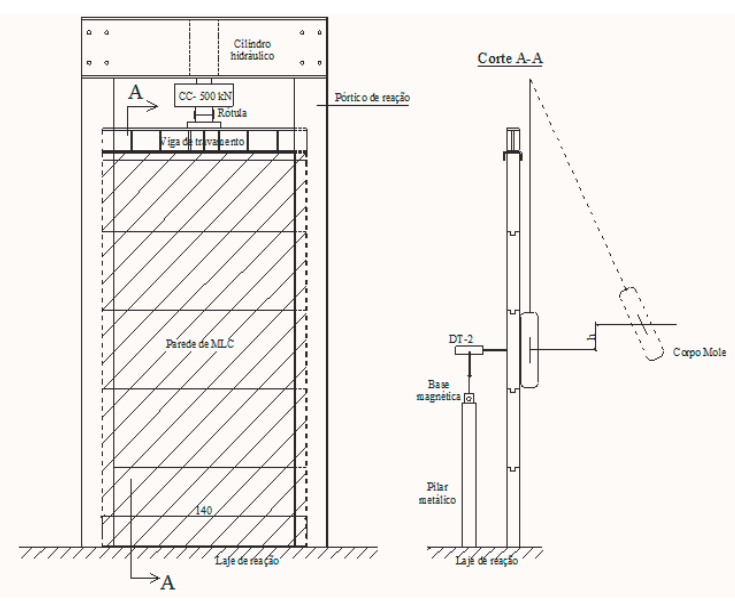

Figura 3 - Ensaio de impacto de corpo mole Fonte: 0 s autores
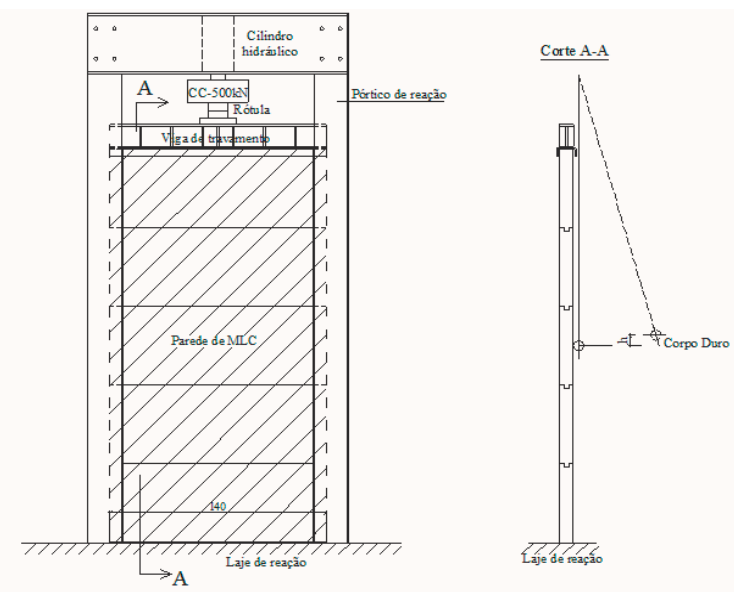

Figura 4 - Ensaio de impacto de corpo duro Fonte: 0 s autores

\section{RESULTADOS E DISCUSSÃO}

\subsection{Caracterização física e mecânica da madeira} Os valores médios das propriedades físicas e mecânicas do Eucalyptus cloeziana e grandis, encontrados nos ensaios, são apresentados na Tabela 2.

\begin{tabular}{|l|l|l|l|l|l|l|}
\hline Espécie & $\begin{array}{l}\mathbf{\rho}_{\mathrm{ap}} \\
(\mathbf{k g} / \mathbf{m} 3)\end{array}$ & $\begin{array}{l}\mathbf{f}_{\mathrm{co}} \\
(\mathbf{M P a})\end{array}$ & $\begin{array}{l}\mathbf{f}_{\mathrm{t0}} \\
(\mathbf{M P a})\end{array}$ & $\begin{array}{l}\mathbf{f}_{\mathrm{tg0}} \\
(\mathbf{M P a})\end{array}$ & $\begin{array}{l}\mathbf{f}_{\mathrm{v}} \\
(\mathbf{M P a})\end{array}$ & $\begin{array}{l}\mathbf{E}_{\mathrm{co}} \\
(\mathbf{M P a})\end{array}$ \\
\hline $\begin{array}{l}\text { Eucalyptus } \\
\text { cloeziana }\end{array}$ & 893 & 59,8 & 94,8 & 5,1 & 11,5 & 13963 \\
\hline $\begin{array}{l}\text { Eucalyptus } \\
\text { grandis }\end{array}$ & 645 & 41,3 & 73,2 & 2,9 & 7,9 & 12813 \\
\hline
\end{tabular}

Tabela 2 - Valores médios das propriedades do Eucalyptus cloeziana e Eucalyptus grandis Fonte: 0 s autores

\subsection{Ensaio de compressão simples}

Os resultados dos ensaios de compressão simples nas paredes de Eucalyptus cloeziana e Eucalyptus grandis são apresentados nas Tabelas 3 e 4, respectivamente. 


\begin{tabular}{|l|l|l|l|l|}
\hline Parede & $\begin{array}{l}\text { Carga } \\
\text { máxima } \\
\text { aplica- } \\
\text { da (kN) }\end{array}$ & $\begin{array}{l}\text { Flecha (1) } \\
\text { máxima } \\
\text { (mm) }\end{array}$ & $\begin{array}{l}\text { Deslocamentos } \\
\text { vertical (en- } \\
\text { curtamento) } \\
\text { máximo (mm) }\end{array}$ & $\begin{array}{l}\text { Inclinação do } \\
\text { gráfico carga } \\
\text { xdeslocamen- } \\
\text { to (kN/mm) }\end{array}$ \\
\hline A & 350 & 0,97 & 6,88 & 54,599 \\
\hline B & 350 & 0,95 & 9,16 & 54,229 \\
\hline C & 350 & 2,31 & 10,24 & 45,788 \\
\hline
\end{tabular}

Tabela 3 - Resultados dos ensaios de compressão simples nas paredes de Eucalyptus cloeziana Fonte: 0 s autores

\begin{tabular}{|l|l|l|l|l|}
\hline Parede & $\begin{array}{l}\text { Carga } \\
\text { máxima } \\
\text { aplica- } \\
\text { da (kN) }\end{array}$ & $\begin{array}{l}\text { Flecha (1) } \\
\text { máxima } \\
\text { (mm) }\end{array}$ & $\begin{array}{l}\text { Deslocamentos } \\
\text { vertical (en- } \\
\text { curtamento } \\
\text { máximo } \mathbf{~ m m ) ~}\end{array}$ & $\begin{array}{l}\text { Inclinação do } \\
\text { gráfico carga } \\
\text { x deslocamen- } \\
\text { to (kN/mm) }\end{array}$ \\
\hline A & 200 & 0,87 & 13,22 & 16,030 \\
\hline B & 244 & 1,30 & 21,00 & 16,190 \\
\hline
\end{tabular}

Tabela 4 - Resultados dos ensaios de compressão simples nas paredes de Eucalyptus grandis Fonte: 0 s autores

Analisando as Tabelas 3 e 4 percebe-se que as paredes de Eucalyptus cloeziana se comportaram melhor, resistiram a maiores cargas, tiveram menores deslocamentos e, consequentemente, apresentaram maiores rigidezes. Na Figura 5 é apresentado um gráfico representativo do comportamento das paredes nos ensaios de compressão simples e na Figura 6 é apresentado um gráfico com as regressões lineares para obter o modulo de elasticidade da parede $\left(E_{p}\right)$. A Figura 5 e 6 referem-se à parede de Eucaliptus cloeziana.

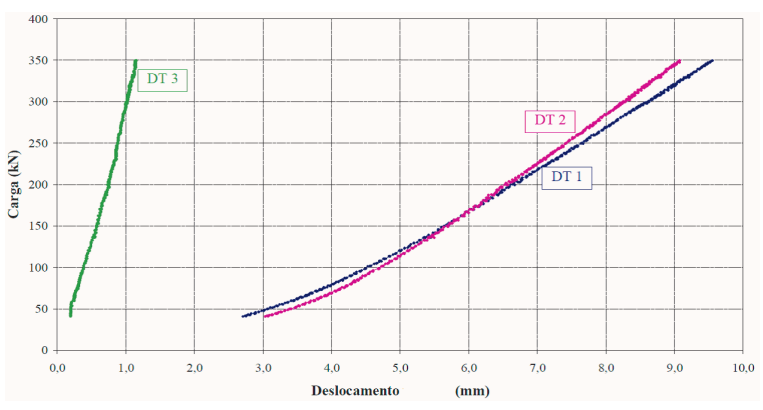

Figura 5 - Compressão Parede B, Eucaliptus cloeziana - Carga x deslocamento Fonte: 0 s autores

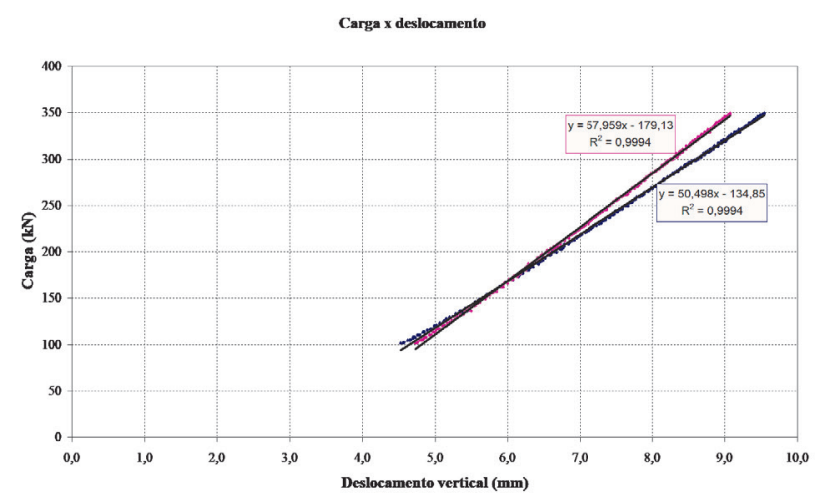

Figura 6 - Compressão Parede B, Eucaliptus cloeziana - Carga x encurtamento, (Ep) Fonte: 0 s autores

\subsection{Ensaios de impacto de corpo mole e de cor- po duro}

Como resultados dos ensaios de impacto de corpo mole são apresentados os seguintes deslocamentos: horizontal instantâneo $\left(d_{h}\right)$, obtido pelo DT no momento do impacto; o deslocamento horizontal residual $\left(\mathrm{d}_{\mathrm{hr}}\right)$, obtido pelo DT após o impacto e passado o período de estabilização da parede. Os resultados são apresentados na Tabela 5.

Os resultados dos ensaios de impacto de corpo duro são apresentados na Figura 6. Devido a limitações de altura no laboratório não foram realizados os ensaios com energia de impacto de $720 \mathrm{~J}$, assim este valor não foi apresentado na tabela, apesar de ser previsto na norma NBR 15575 (2013).

Uma análise dos critérios de desempenho estabelecidos para o ensaio de corpo mole (NBR 15575, 2013) e dos resultados obtidos nas paredes de MLC indicam o excelente desempenho. As paredes de Eucalyptus cloeziana se comportaram melhor do que as paredes de Eucalyptus grandis. Os deslocamentos máximos obtidos nos ensaios estão dentro dos limites estabelecidos e também não houve grandes danos, apenas leves escamações superficiais nos ensaios com a maior energia de impacto.

\begin{tabular}{|c|c|c|c|c|c|}
\hline \multirow[t]{2}{*}{ Parede } & \multirow[t]{2}{*}{ Energia (J) } & \multicolumn{2}{|l|}{ Eucalyptus cloeziana } & \multicolumn{2}{|l|}{ Eucalyptus grandis } \\
\hline & & Instantâneo - $d_{h}(\mathrm{~mm})$ & Residual - $d_{h r}(\mathrm{~mm})$ & Instantâneo - $d_{h}(\mathrm{~mm})$ & Residual - $d_{h r}(\mathrm{~mm})$ \\
\hline \multirow[t]{7}{*}{ A } & 60 & 1,8 & 0,3 & 3,1 & 0,2 \\
\hline & 120 & 4,5 & 0,6 & 6,7 & 0,2 \\
\hline & 180 & 6,3 & 0,6 & 8,8 & 0,2 \\
\hline & 240 & 9,4 & 0,4 & 13,9 & 0,4 \\
\hline & 360 & 13,8 & 0,2 & 20,8 & 0,6 \\
\hline & 480 & 16,9 & 0,3 & 23,9 & 0,6 \\
\hline & 600 & 22,0 & 0,2 & 30,3 & 0,6 \\
\hline \multirow[t]{2}{*}{ B } & 60 & 2,0 & 0,1 & 2,6 & 0,3 \\
\hline & 120 & 3,0 & 0,1 & 6,5 & 0,3 \\
\hline
\end{tabular}




\begin{tabular}{|c|c|c|c|c|c|}
\hline \multirow[t]{5}{*}{ B (continuação) } & 180 & 4,4 & 0,1 & 9,1 & 0,3 \\
\hline & 240 & 5,5 & 0,4 & 8,1 & 0,3 \\
\hline & 360 & 7,2 & 0,2 & 11,2 & 0,3 \\
\hline & 480 & 9,0 & 0,2 & 14,7 & 0,3 \\
\hline & 600 & $10,0^{(1)}$ & $0,3^{(1)}$ & 19,4 & 0,3 \\
\hline \multirow[t]{7}{*}{ C } & 60 & 1,5 & 0,3 & \multirow{2}{*}{\multicolumn{2}{|c|}{ (1) Pequena escamação na região do impacto (ver Figura) }} \\
\hline & 120 & 2,6 & 0,2 & & \\
\hline & 180 & 3,9 & 0,2 & & \\
\hline & 240 & 7,1 & 0,2 & & \\
\hline & 360 & 8,7 & 0,1 & & 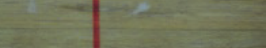 \\
\hline & 480 & 10,2 & 0,2 & & \\
\hline & 600 & 13,6 & 0,2 & & \\
\hline
\end{tabular}

Tabela 5 - Resultados dos deslocamentos medidos nos ensaios de impacto de corpo mole Fonte: 0 s autores

A NBR 15575 (2013) prescreve as alturas de queda (h) e os critérios de desempenho para os ensaios de impacto de corpo duro. Os impactos com energias de 2,5 J e $10 \mathrm{~J}$ servem para a avaliação de paredes estruturais na parte interna das habitações e aqueles com energias de 3,75 J e $20 \mathrm{~J} \mathrm{servem} \mathrm{para} \mathrm{as} \mathrm{partes} \mathrm{externas.} \mathrm{As} \mathrm{profundidades} \mathrm{das}$ mossas medidas nos ensaios de corpo duro, para todas as paredes e para todas as energias sendo para cada energia 10 ensaios, são apresentadas na Figura 7.

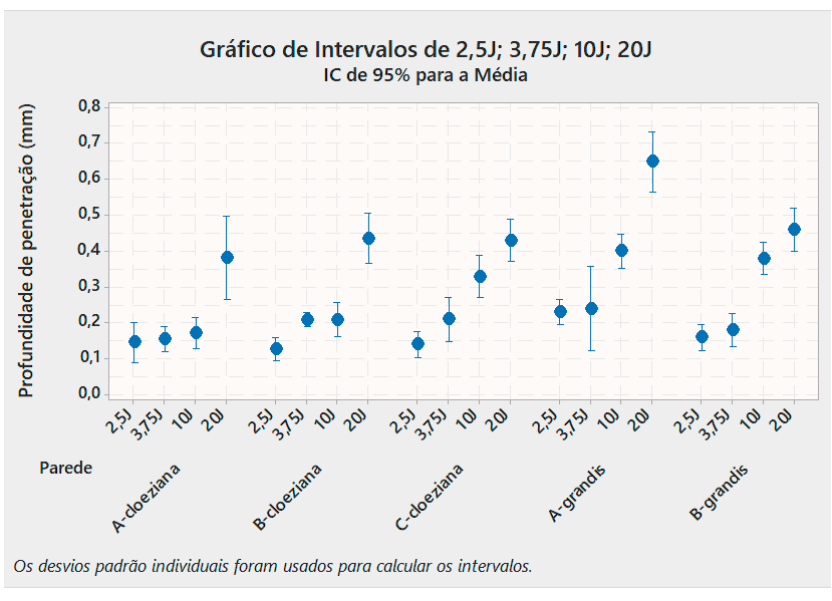

Figura 7 - Resultados ensaios corpo duro - Energia de impacto x profundidade de penetração Fonte: 0 s autores

Analisando os resultados obtidos, pode-se dizer que as profundidades das mossas medidas nos ensaios foram muito inferiores aos limites estabelecidos pela NBR 15575 (2013). Nos ensaios com a esfera de $1000 \mathrm{~g}$ não foram observados fissuras ou traspassamentos em nenhuma das paredes ensaiadas. Exemplos das mossas deixadas nas paredes após os ensaios com esferas de $1000 \mathrm{~g}$ são apresentados na Figura 8.

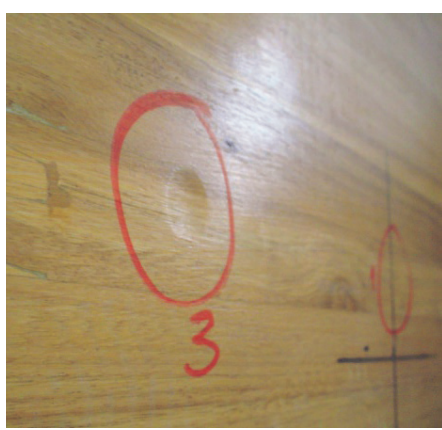

(a)

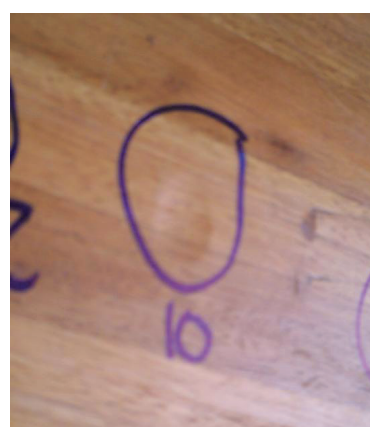

(b)
Figura 8 - Mossa no ensaio com esfera de $1000 \mathrm{~g}$ : (a) Altura de queda de $100 \mathrm{~cm}$ (b) Altura de queda de $200 \mathrm{~cm}$

Fonte: 0 s autores

\section{CONCLUSÕES}

As paredes de Eucalyptus cloeziana se comportaram meIhor do que as paredes de Eucalyptus grandis. Não houve grandes danos, apenas leves escamações superficiais nos ensaios com maior energia de impacto. A partir dos critérios estabelecidos para os ensaios de impacto de corpo mole (condições para dh e dhr) na NBR15575 (2013) pode-se afirmar que o desempenho das paredes de MLC pode ser classificado como Intermediário ou Superior.As profundidades das medidas nos ensaios de impacto de corpo duro foram muito inferiores aos limites estabelecidos pela NBR15575 (2013). Nos ensaios com a esfera de $1000 \mathrm{~g}$ não foram observadas fissuras ou traspasses em nenhuma das paredes ensaiadas. Pode-se afirmar quanto às análises dos resultados dos ensaios de impacto de corpo duro que o desempenho das paredes de MLC pode ser classificado como Intermediário ou Superior. 


\section{AGRADECIMENTOS}

Ao Conselho Nacional de Desenvolvimento Científico e Tecnológico (CNPq) e à Fundação de Amparo à Pesquisa do Estado de Minas Gerais (FAPEMIG), pelo apoio financeiro concedido a esta pesquisa.

\section{REFERÊNCIAS}

Associação Brasileira da Indústria de Madeira Processada Mecanicamente; “Estudo setorial 2004, indústria de madeira processada mecanicamente". Curitiba, Paraná, 52 p.,2004.

AMORIM, S.T.A.; MANTILLA, J.N.R.; CARRASCO, E.V.M.; "A madeira laminada cruzada: aspectos tecnológicos, construtivos e de dimensionamento" Revista Matéria, suplemento, e-11937, p. 1-7, 2017.

ASSOCIAÇÃO BRASILEIRA DE NORMAS TÉCNICAS, ABNT,NBR 7190 - Projeto de estruturas de madeira, Rio de Janeiro,1997, 107 p.

ASSOCIAÇÃO BRASILEIRA DE NORMAS TÉCNICAS, ABNT, NBR 15575 - Edificações habitacionais, Rio de Janeiro,2013, 115 p.

BALDWIN, R.F.; "Plywood and veneer-based products: manufacturing practices". San Francisco: M. Freeman, 388 p., 1995.

BUILDING AND CONSTRUCTION AUTHORITY;“Design for manufacturing and assembly (DfMA), Mass Engineered Timber (MET)" version 1.0, Guidebook, 75 p., 2018.

CABRAL C.P.T.; VITAL, B.R.; DELLA LUCIA, R.M.; PIMENTA, A.S.; SOARES, C.P.B.; CARVALHO, A.M.M.L.; "Propriedades de chapas tipo OSB, fabricadas com partículas acetiladas de madeiras de Eucalyptus grandis, Eucalyptus urophylla, Eucalyptus cloezianae Pinus elliottii" R. Árvore, Viçosa-MG, v.30, n.4, p.659-668,2006.

IWAKIRI, S.; KEINERT JUNIOR, S.;PRATA,J.G.;ROSSO, S.;"Produção de painel compensado estrutural de Eucalyptus grandis e Eucalyptus dunnii" FLORESTA, v. 37, n. 3, p. 363-367,2007.

IWAKIRI, S.; ANDRADE, A.S.; CARDOSO JUNIOR, A.A.; CHIMPANSKI,E.R.; PRATA, J.G.; ADRIAZOLA, M.K.O.;"Produção de painéis aglomerados de alta densificação com uso de resina melaminauréiaformaldeído" Revista Cerne, v.11, p.323-328,2005.

LIMA, D.C.; MELO,R.R.; PIMENTA, A.S.; PEDROSA, T.D.; SOUZA, M.J.C.; SOUZA, E.C.; "Physical-mechanical properties of wood panel composites produced with Qualea sp. sawdust and recycled polypropylene" Environmental Science and Pollution Research, v.
27, p. 4858-4865, 2020.

MACEDO, B.; FERRO, F.S.; VARANDA, L.D.; CAVALHEIRO, R.S.; CHRISTOFORO, A.L.; LAHR, F.A.R.; "Propriedades físicas de painéis aglomerados de madeira produzidos com adição de película de polipropileno biorientado"Revista Brasileira de Engenharia Agrícola e Ambiental, v.19, n.7, p.674-679,2015.

MAHR, K.; SINHA, A.; BARBOSA, A.R.; “Experimental Investigation and Modeling of Thermal Effects on a Typical Cross-Laminated Timber Bracket Shear Connection" J. Mater. Civ. Eng., v. 32, n. 6, p, 04020111 , 2020.

SIKORA,K.S.;MCPOLIN,D.O.;HARTE, A.M.; "Effects of the thickness of cross-laminated timber (CLT) panels made from Irish Sitka spruce on mechanical performance in bending and shear" Construction and Building Materials, v. 116, p. 141-150, 2016. 


\section{AUTORES}

ORCID: 0000-0001-7870-0283

EDGAR VLADIMIRO MANTILLA CARRASCO, Dr. | Universidade de São Paulo, Campus São Carlos | Doutorado em Estruturas | São Carlos, SP - Brasil | Correspondência para: R. Ouro Preto 1240/1201 - Santo Agostinho, Belo Horizonte - MG, 30170-048 | E-maill: mantilla.carrasco@gmail.com

ORCID: 0000-0001-9510-2859

CYNARA FIEDLER BREMER, Dra. | Universidade Federal de Minas Gerais | Doutorado em Engenharia de Estruturas | Belo Horizonte, MG - Brasil | Correspondência para: R. Paraíba, 697 - Funcionários, Belo Horizonte - MG, 30130-141 | E-maill: cynarafiedlerbremer@ufmg.br

ORCID: 0000-0001-7426-0970

JUDY NORKA RODO DE MANTILLA, Dra. | Universidade de São Paulo, Campus São Carlos | Doutorado em Engenharia Civil - Geotecnia | São Carlos, SP - Brasil | Correspondência para: R. Ouro Preto 1240/1201 - Santo Agostinho, Belo Horizonte - MG, 30170-048 | E-maill: judynorka@gmail. com

\section{COMO CITAR ESTE ARTIGO}

CARRASCO, Edgar Vladmiro Mantilla; BREMER, Cynara Fiedler; MANTILLA, Judy Norka Rodo de. Avaliação do Desempenho Estrutural de Paredes de Madeira Laminada Colada. MIX Sustentável, [S.I.], v. 6, n. 3, p. 83-90, jun. 2020. ISSN 24473073. Disponível em:<http://www.nexos.ufsc.br/index.php/mixsustentavel>. Acesso em: dia mês. ano. doi:https://doi. org/10.29183/2447-3073.MIX2020.v6.n3.83-90. 